\title{
El sentimiento de soledad en personas mayores: conocimiento y tamización oportuna
}

\section{Loneliness in Older People: Timely Knowledge and Screening}

Recepción: 06 Junio 2019 | Aceptación: 02 Agosto 2019

\section{Claudia Marcela Camargo-Rojas ${ }^{a}$}

Enfermera. Magíster en Epidemiología. Docente del Departamento de Enfermería en Salud Colectiva de

la Facultad de Enfermería, Pontificia Universidad Javeriana, Bogotá, Colombia

Diego Andrés Chavarro-Carvajal Médico especialista en Medicina Interna. Especialista en Geriatría. Magíster en Epidemiología. Candidato a Doctorado en Investigación en Gerontología. Docente

del Instituto de Envejecimiento de la Facultad de Medicina, Pontificia Universidad Javeriana y de la Unidad de Geriatría del Hospital Universitario San Ignacio, Bogotá, Colombia

a Autora de correspondencia:
camargo.claudia@javeriana.edu.co

Cómo citar: Camargo Rojas CM, Chavarro Carvajal DA. El sentimiento de soledad en personas mayores: conocimiento y tamización oportuna. Univ. Med. 2020;61(2). https://doi.org/10.11144/Javeriana.umed 61-2.essm

\section{RESUMEN}

El sentimiento de soledad tiene una prevalencia del $40 \%$ en las personas mayores, secundario a situaciones de abandono, duelo y adaptación del rol. Se ha estudiado su impacto en el desarrollo de condiciones crónicas, disminución del autocuidado y adherencia al tratamiento. Los factores asociados con esta condición son edad avanzada, ser mujer, ausencia de pareja y un bajo nivel educativo. El propósito de esta revisión es resaltar la importancia de la inclusión de la valoración rutinaria de la soledad en personas mayores con instrumentos validados con el fin de generar intervenciones enfocadas en este fenómeno. En población colombiana se cuenta con una escala validada para su tamización, la cual debe ser considerada en el momento de la atención y seguimiento de las personas mayores por los profesionales de la salud.

Palabras clave

soledad; persona mayor; envejecimiento poblacional.

\section{ABSTRACT}

Loneliness has a prevalence of $40 \%$ in older people secondary to situations of abandonment, bereavement and adaptation of the role. The impact it has on the development of chronic conditions, decreased self-care and adherence to treatment has been studied. The factors associated with this condition are advanced age, being a woman, absence of a partner and a low educational level. The purpose of this review is to highlight the importance of the inclusion of routine assessment of loneliness in older people with validated instruments in order to generate interventions focused on this phenomenon. In the Colombian population, there is a validated scale for screening which should be considered at the time of care and monitoring of the elderly by health professionals. 
Keywords

loneliness; aged; aging population.

\section{Introducción}

Cuando se habla del envejecimiento poblacional, se resalta el interés de abarcar este tema en el ámbito de salud, debido a que nos corresponde dar respuesta a las nuevas necesidades de la dinámica demográfica y al aumento de la demanda de servicios de salud por parte del adulto mayor $(1,2)$. De acuerdo con el boletín de envejecimiento de la Organización Mundial de la Salud, en el 2050 el número de personas mayores de 65 años de edad será superior al de niños menores de 14 años (3).

Cabe mencionar que la carga de enfermedad del adulto mayor es elevada por todas aquellas enfermedades crónicas no transmisibles, como hipertensión arterial, diabetes mellitus, enfermedad ateroesclerótica, entre otras $(4,5)$. Sin embargo, se evidencia un aumento de la carga de las enfermedades mentales, a causa de todos los cambios y adaptaciones que debe generar el adulto mayor en este ciclo de vida (6).

Dentro del espectro de los cambios reconocidos en la persona mayor está el sentimiento de soledad, considerado un predictor de ansiedad y depresión, al igual que del desarrollo de otras condiciones en salud, tal como se demuestra en el estudio de Bekhet y Zauszniewski (7), donde se relacionó el aumento en las cifras de tensión arterial consecuente de la elevación de cortisol con la soledad como factor estresor.

Vicente Madoz Jáuregui (8) ha descrito la soledad como el convencimiento de ser excluido, de no presentar interacciones y carecer de compañía para el desarrollo de las actividades. Este factor biopsicosocial, como es considerado, se clasifica en dos tipos: objetivo (sentimiento agradable) y subjetivo (sentimiento desagradable), así como en soledad conyugal, familiar, social y crisis existencial, como lo describe la escala ESTE (9), validada y adaptada para medir el sentimiento de soledad en población adulta mayor colombiana, con un $\alpha$ de Cronbach 0,90 (10,11).
En la revisión sistemática realizada por Petitte et al. (12), donde se incluyeron artículos en inglés de enfoque cuantitativo con rigor metodológico, y fue seleccionado solamente un artículo de Suramérica, se evidenció que el sentimiento de soledad es un desencadenante para el desarrollo de enfermedades crónicas, como: enfermedad cardiaca, hipertensión arterial, enfermedad cerebrovascular, enfermedad pulmonar, enfermedad metabólica, entre otras. Cabe mencionar que en la biblioteca Cochrane no se encuentran revisiones sobre este tema.

En relación con la población colombiana, diversas investigaciones se han enfocado en reconocer el fenómeno de la soledad desde su magnitud hasta los diferentes factores que predisponen su desarrollo, así como la validación y adaptación de escalas que permitan una valoración objetiva $(10,13,14)$.

Otros estudios se han centrado en la depresión de la persona mayor; pero, como lo menciona Theeke (15), la soledad es un constructo psicológico separado de la depresión, por lo que se debe considerar seguir investigando en torno a esta problemática, especialmente sobre su impacto para el desarrollo de condiciones crónicas en salud. Esta revisión de tema pretende resaltar la importancia de incluir en la valoración rutinaria de la soledad en personas mayores instrumentos validados con el fin de generar intervenciones enfocadas en este fenómeno.

\section{Epidemiología del envejecimiento poblacional}

Dentro del proceso y dinámica poblacional en la que el mundo se encuentra, cuando se está incrementando el número de personas mayores, aumentando la esperanza de vida y, a su vez, produciendo cambios en la transición demográfica, se está despertando el interés de continuar indagando sobre los nuevos desafíos, en búsqueda de herramientas que permitan seguir construyendo condiciones dignas en la población mayor.

De acuerdo con el Centro Latinoamericano y Caribeño de Demografía de la Comisión 
Económica para América Latina, en los países de la región de América Latina y el Caribe, la población mayor de 60 años aumentará, reflejado en un crecimiento mayor en comparación con otros grupos etarios y de tres a cinco veces más en la población total entre los años 2025 y 2050 (16). En relación con la población colombiana, y según el último censo (año 2005) del Departamento Administrativo Nacional de Estadística, se evidenció que la población mayor de 65 años representaba un 6,5\%, y se proyecta al 2050 un aumento en esta población del $20 \%$ (17).

El envejecimiento se define como el proceso que se da desde el nacimiento hasta la muerte, pues durante todo el trayecto de vida se genera crecimiento y desarrollo, abarcando los aspectos biológicos, psicosociales y espirituales (18). Estas dimensiones deben abordarse desde las políticas públicas, para las cuales la vejez se convierte en un evento de interés en salud pública, con el fin de garantizar la optimización del potencial de las personas por la experiencia de vida, la minimización de los riesgos en salud, la promoción de la autonomía y el mejoramiento de la calidad de vida (19).

La mortalidad en la población mayor colombiana se atribuye a cardiopatía isquémica, enfermedad cerebrovascular y enfermedad pulmonar obstructiva crónica, según un estudio de carga de enfermedad (6). De igual forma, se evidenció en la Encuesta de Salud, Bienestar y Envejecimiento (SABE) que respecto a la morbilidad, el 84,8 \% de la población padece más de una condición crónica (pluripatológicos), aun cuando sobresalen dos condiciones crónicas en más del 40 \%: hipertensión y síntomas depresivos. Estos son característicos a mayor edad y en estratos socioeconómicos altos (20).

Frente a la dinámica de la población mayor, se han creado centros de encuentro de la persona mayor o centros día donde sin institucionalizar a un individuo se le permite la interacción. Ello crea redes de apoyo, seguridad económica, así como promoción de asistencia social frente a la soledad.

\section{Soledad y adulto mayor}

El sentimiento de soledad representa una prevalencia del $20 \%$ al $40 \%$ en la población adulta mayor (12), una situación desfavorable que puede traerle repercusiones negativas. Algunos autores lo describen como falta de intercambio con el otro, a partir de lo cual se generan sentimientos de tristeza, miedo, ansiedad, que en las personas mayores puede considerarse una imposición difícil de abordar $(10,21)$.

El aumento de la prevalencia de soledad es representativo de las personas mayores, pues aparecen condiciones, por ejemplo, pérdida, abandono, duelo, cambio de rol, que pueden contribuir a la aparición de esta, tal como lo menciona Rodríguez Martín (22), para quien existen factores causales de la soledad, como crisis de identidad, de autonomía y pertenencia.

Cabe establecer la diferencia entre estar solo y sentirse solo. Autores como Rubio y Aleixandre (9) y Yaben (23) destacan que es importante diferenciar entre ambos conceptos, pues lo primero se asocia con aislamiento social y carencia de redes sociales (soledad objetiva) y el segundo lo definen como soledad subjetiva o emocional.

Las consecuencias de la soledad se pueden desglosar desde las dimensiones física, psicológica y social. En la primera se puede presentar debilidad del sistema inmunológico, problemas cardiacos o digestivos y problemas en el patrón del sueño; en la dimensión psicológica, baja autoestima, depresión o alcoholismo y suicidio, y en la dimensión social, prejuicios sociales o aislamiento $(12,22)$. La soledad incrementa en personas mayores no institucionalizadas a medida que aumenta la edad (14), y de acuerdo con las implicaciones en la vejez, adquiere relevancia la creación de programas e intervenciones de seguimiento para la prevención de la soledad y el mantenimiento de la salud de este grupo poblacional (24). 


\section{Factores asociados a la soledad}

En dos estudios realizados $(15,25)$ se encontró que las variables sociodemográficas como edad, estado civil, estrato socioeconómico y nivel educativo se asocian con la aparición del sentimiento de soledad; de igual forma, la calidad de las redes sociales, depresión y falta de afiliación religiosa. Una de la primeras variables asociadas es la edad, puesto que a mayor edad, mayor sentimiento de soledad, tal como quedó evidenciado en un estudio realizado Graneheim y Lundman (26), en el cual las personas de 85 a 103 años se sentían solas a causa de la muerte de la pareja, de familiares y de amigos.

En relación con el género, varios estudios refieren que es mayor el sentimiento de soledad en las mujeres $(13,15)$, pues estas les dan mayor importancia a las pérdidas que ocurren en este transcurrir vital, aparte de que están expuestas a la viudez y al abandono por parte de los hijos. Por otra parte, el estrato socioeconómico se encuentra como un factor asociado, debido a la reducción aparente de ingresos económicos y a falta de un seguro de pensión de la vejez, que pone en riesgo el bienestar social. Se establece que a mayor nivel socioeconómico se genera un aumento de la red social (13).

Frente al nivel educativo (25), un mayor nivel de escolaridad es considerado un factor protector para la aparición del sentimiento de soledad, aunque el estudio de Rubio y Aleixandre (9) encontró que el $25 \%$ de quienes autoperciben soledad presentan estudios medios o superiores.

\section{Efectos de la soledad en la enfermedad crónica no transmisible}

Diferentes estudios han generado el interrogante sobre el efecto que el sentimiento soledad puede generar en la condición de salud de las personas mayores, especialmente en el desarrollo o en el no control o exacerbación de los síntomas de las enfermedades crónicas no transmisibles.

En las enfermedades cardiovasculares, según el estudio Hackett et al. (27), se halló una asociación con el sentimiento de soledad, debido a los mecanismos hormonales derivados del estrés, lo que aumenta la presión arterial por la resistencia vascular periférica y la respuesta inflamatoria, observándose principalmente en las mujeres. En el estudio realizado por Theeke y Mallow (28) se reportó en una muestra de 60 participantes que la soledad prevalece en población con enfermedades crónicas, y que la soledad era mayor en población con enfermedad cardiaca, con un $68 \%(n=41)$.

Por otro lado, se encuentra el efecto del sentimiento de soledad en la generación de síndrome metabólico o diabetes mellitus, tal como se refleja en un estudio realizado en Inglaterra, donde se midió la circunferencia de cintura, la tensión arterial, el perfil lipídico y la glucosa y se encontró una estrecha asociación entre soledad y síndrome metabólico con un OR de 1,1 (IC 95\%: 1,0-1,2), resultado de una alteración en la producción de cortisol, activación del sistema simpático y producción de insulina (29).

El efecto de soledad en la enfermedad pulmonar se evidencia en un $67 \%$ de la población del estudio de Keele-Card et al. (30), en el cual, por medio de la medición del sentimiento de soledad realizada con la escala de UCLA, se encontró un sentimiento moderado de soledad en pacientes con enfermedad obstructiva crónica. Datos similares encontrados en otro estudio realizado en Turquía señalaron que el 97 $\%$ de los participantes tenía el mismo grado de soledad, y resaltaron una asociación significativa entre la presencia de redes de apoyo y la disminución del sentimiento (31).

\section{Soledad y funcionalidad}

Así como se ha mencionado la relación entre el sentimiento de soledad, el mal control de enfermedades crónicas, la mala autopercepción de salud y la depresión, el estado funcional es un marcador crucial del estado de salud en el anciano (32), y su afectación representa deterioro en el estado de salud. Existen estudios que vinculan la soledad con la pérdida de función, al igual que de la asociación entre soledad y 
compromiso funcional $(15,33)$. De este modo, el estado funcional debe considerarse una potencial vía de eventos adversos relacionados con la soledad, y a su vez asociado con el incremento en la mortalidad por todas las causas $(32,34)$. El sentimiento de soledad se puede relacionar con limitación en el espacio de vida, lo que aumenta el riesgo de inactividad física, al igual que deterioro funcional progresivo y mortalidad (34).

\section{Escalas de medición del sentimiento de soledad}

En la literatura sobre el tema se encuentran diferentes instrumentos de medición de la soledad (24), por ejemplo: la escala UCLALonelines Scale, creada por Russell et al., en 1980, que es una de las más utilizadas para medir el sentimiento de soledad y se encuentra compuesta por dos aspectos: intimidad con otros y sociabilidad, con 20 ítems tipo Likert; la escala Social and Emotional Loneliness Scale for Adults (SESLA), conformada por tres subescalas: romántica o de pareja, relaciones con la familia y relaciones con los amigos; la escala Emotional Social Loneliness Inventory (ESLI), que evalúa dos categorías: soledad emocional y soledad social, y la Escala de Satisfacción Vital de Philadelphia, basada en que el bienestar psicológico parte del estado de ánimo elevado y satisfacción personal $(10,11,24)$.

A partir de las escalas anteriores, Ramona Rubio y Manuel Aleixandre $(9,35)$ construyeron la escala ESTE, validada en 295 personas mayores de 65 años. Se encontró que esta permite evaluar todos los constructos de soledad, generándose las categorías de soledad familiar, soledad conyugal, soledad social y crisis de adaptación o soledad existencial, el $\alpha$ de Cronbach fue de 0,92, con un total de 34 ítems.

En Colombia, los autores Cardona et al. (10) y Cerquera et al. (11) validaron la escala ESTE en población colombiana; los primeros en población del municipio de Bello (Antioquia), con una muestra de 180 adultos mayores de 55 años ( $\alpha$ de Cronbach de 0,90), y los segundos, en
Bucaramanga (Santander), con una muestra 383 personas mayores de 65 años ( $\alpha$ de Cronbach de 0,92 ). Ambas investigaciones validaron 30 ítems de la escala original.

\section{Intervención gerontológica para la soledad}

Diferentes autores han presentado actividades multidisciplinares basadas en seis factores especiales para enfrentar el reto del sentimiento de soledad: el fomento de la actividad física, masoterapia, musicoterapia, rehabilitación, actividades lúdicas y aromaterapia (36). Estas actividades se pueden realizar según los contextos locales y deben estar ajustadas a la realidad de cada población y país.

Adicionalmente, las estrategias usadas deben partir de evaluar el problema, a fin de crear programas terapéuticos individualizados de prevención y control de la soledad. También proponer soluciones que tengan en cuenta al individuo, así como soluciones colectivas que incluyen a la familia (cuando se cuenta con ella), recursos sociales (por ejemplo, centros día, grupos vecinales y actividades de esparcimiento), evaluación por profesionales de la salud, relaciones de vecindad y vínculo a programas de educación del sistema educativo (22).

Tal como lo mencionan Bermeja y Ausin (24), a partir de una revisión sistemática de la literatura sobre programas para combatir la soledad, las actividades deben enfocarse en brindar espacios donde la persona mayor pueda interactuar con otros, por medio de actividades de ocupación humana como: talleres de jardinería/horticultura, terapia de humor y programas de ejercicio físico; así como fortalecer redes familiares y sociales por medio de videoconferencias, taller de reminiscencia e intervención con animales. En los espacios institucionalizados se recomiendan actividades grupales para fortalecer la comunicación y contactos sociales. Se resalta que estos programas no solamente se centran en la prevención de la soledad, sino en la calidad de vida, control y mantenimiento de la salud y bienestar psicológico (7). 


\section{Conclusiones}

El sentimiento de soledad es frecuente en la población adulta mayor, y aún más en personas en situaciones desfavorables. Usualmente, está asociado con variables sociodemográficas, como mayor edad, ser mujer, ausencia de pareja y bajo nivel educativo. Adicionalmente, está asociado con la presencia de enfermedades crónicas. Se cuenta con escalas de medición para su tamización, incluso con validación realizada en población colombiana, por lo cual se sugiere incluir la soledad como aspecto importante en la valoración integral gerontológica en el momento de la atención y seguimiento de personas mayores por parte de los profesionales de la salud.

De igual forma, se recomienda considerar programas de prevención de la soledad con mejores metodologías de abordaje, diferentes a la mencionadas, que promocionen un envejecimiento saludable no solamente en la vejez, sino en edades tempranas; así como actividades que contribuyan a mejorar la capacidad funcional, la percepción propia y el mantenimiento de la salud en escenarios de integración y escucha activa.

\section{Referencias}

1. Segura A, Cardona E, Segura A, Garzón M. Riesgo de depresión y factores asociados en adultos mayores. Antioquia, Colombia. Rev Salud Pública. 2015;17(2):184-94.

2. Rivillas JC, Gómez-Aristizábal L, Rengifo-Reina HA, Muñoz-Laverd EP. Envejecimiento poblacional y desigualdades sociales en la mortalidad del adulto mayor en Colombia: ipor qué abordarlos ahora y dónde comenzar? Rev Dac Nac Salud Pública. 2017;35(3):369-81. https://doi.org/10. 17533/udea.rfnsp.v35n3a07.

3. Organización Mundial de la Salud (OMS). Informe mundial sobre el envejecimiento y la salud. J Chem Inf Model. 2015;53(9):1689-99.
4. Montes Santiago J, Casariego Vales E, de Toro Santos M, Mosquera E. La asistencia a pacientes crónicos y pluripatológicos. Magnitud e iniciativas para su manejo: La Declaración de Sevilla. Situación y propuestas en Galicia. Galicia Clín. 2012;73(1):7-14.

5. Colombo PB, Nieto Martín MD, De La Pisa BP, Lozano MJG, Camiez MAO, Wittel MB. Validación de un modelo pronóstico para pacientes pluripatológicos en atención primaria: estudio PROFUND en atención primaria. Aten Primaria. 2014;46(S3):41-8.

6. Peñaloza R, Salamanca N, Rodríguez J, Rodríguez J, Beltrán A. Estimación de la carga de enfermedad para Colombia. Bogotá: Editorial Pontificia Universidad Javeriana; 2014.

7. Bekhet A, Zauszniewski J. Mental health of elders in retirement communities: Is loneliness a key factor? Am J Nurs. 2012;26(3):214-24.

8. Madoz Jáuregui V. 10 palabras clave sobre los miedos del hombre moderno. Estella, Navarra: Verbo Divino; 1998.

9. Rubio R, Aleixandre M. La escala "ESTE", un indicador objetivo de soledad en la tercera edad. Geriátrika. 1999;15(9):26-35.

10. Cardona Jiménez JL, Villamil Gallego MM, Henao Villa E, Quintero Echeverri Á. Validación de la escala para medir la soledad de la población adulta. Investig Educ Enferm [internet]. 2010;28(3):416. Disponible en: http://www.redalyc.org/articulo.oa? $\mathrm{id}=105215721012$

11. Cerquera Córdoba AM, Cala Rueda ML, Galvis Aparicio MJ. Validación de constructo de la escala ESTE-R para medición de la soledad en la vejez en Bucaramanga, Colombia. Divers Perspect Psicol [internet]. 
2013;9(1):45-53. https://doi.org/10.15 332/s1794-9998.2013.0001.03.

12. Petitte T, Mallow JA, Barnes ER, Petrone A, Barr T, Theeke LA. A Systematic review of loneliness and common chronic physical conditions in adults. Open Psychol J. 2015;8(Suppl 2):113-32.

13. Cardona Jiménez JL, Villamil Gallego MM, Henao Villa E, Quintero Echeverri Á. Variables asociadas con el sentimiento de soledad en adultos que asisten a programas de la tercera edad del municipio de Medellín. Med UPB [Internet]. 2015;34(2):102-Disponible en: https://revistas.upb.edu.co/index.p hp/Medicina/article/view/6814.

14. Quintero-Echeverri A, VillamilGallego MM, Henao-Villa E C-JJ. Diferencias en el sentimiento de soledad entre adultos institucionalizados y no institucionalizados. Rev Fac Nac Salud Pública. 2018;36(2).

15. Theeke LA. Predictors of loneliness in U.S. adults over age sixtyfive. Arch Psychiatr Nurs [Internet]. 2009;23(5):387. http://dx.doi.org/10.1 016/j.apnu.2008.11.002.

16. Comisión Económica para América Latina y el Caribe. Las personas mayores en América Latina y el Caribe: diagnóstico sobre la situación y las políticas. Síntesis [internet]. Santiago de Chile: Cepal; 2003. Disponible en: https://repositorio.cepal.org/bitstre am/handle/11362/20180/S0390625_es .pdf? sequence $=1$ \&isAllowed $=\mathrm{y}$

17. Arango VE, Ruiz IC. Diagnóstico de los adultos mayores en colombia [internet]. Bogotá: Fundación Saldarriaga Concha; 2005. Disponible en: http://www.sdp.gov.co/s ites/default/files/diag_adul_mayor.pdf.

18. Brigeiro M. "Envejecimiento exitoso" y "tercera edad": problemas y retos para la promoción de la salud. Investig Educ Enferm. 2005;23(1):102-9.

19. Ministerio de Salud y Protección Social. Envejecimiento demográfico. Colombia 1951-2020: dinámica demográfica y estructuras poblacionales. Bogotá; 2013. Disponible en: https://www.minsalud.g ov.co/sites/rid/Lists/BibliotecaDigital/ RIDE/DE/PS/Envejecimiento-demogr afico-Colombia-1951-2020.pdf

20. Ministerio de Salud y Protección Social, Colciencias. Estudio nacional de salud, bienestar y envejecimiento SABE Colombia: resumen ejecutivo. Bogotá; 2015.

21. Bandari R, Khankeh HR, Shahboulaghi FM, Ebadi A, Keshtkar AA, Montazeri A. Defining loneliness in older adults: protocol for a systematic review. Syst Rev. 2019;8(1):26. https://doi.org/10.1186/s 13643-018-0935-y.

22. Rodríguez Martín M. La soledad en el anciano. Gerokomos. 2009;20(4):159-66.

23. Yaben SY. Adaptación al castellano de la Escala para la Evaluación. Int J Psychol Psychol Ther. 2008;1(1):103-16.

24. Bermeja AI, Ausín B. Programs to combat loneliness in the institutionalised elderly: A review of the scientific literature. Rev Esp Geriatr Gerontol [Internet]. 2018;53(3):155. http://dx.doi.org/10.1 016/j.regg.2017.05.006.

25. Bishop AJ, Martin P. The indirect influence of educational attainment on loneliness among unmarried older adults. Educ Gerontol. 2007;33(10):897-917.

26. Graneheim UH, Lundman B. Experiences of loneliness among the 
very old: The Umeå $85+$ project. Aging Ment Heal. 2010;14(4):433-8.

27. Hackett RA, Hamer $M$, Endrighi R, Brydon L, Steptoe A. Loneliness and stress-related inflammatory and neuroendocrine responses in older men and women. Psychoneuroendocrinology [Internet]. 2012;37 (11):1801. http://dx.doi.org/10 $.1016 / j$.psyneuen.2012.03.016

28. Theeke LA, Mallow J. Loneliness and quality of life in chronically ill rural older adults. Am J Nurs. 2013;113(9):28-38. https://doi.org/10. 1097/01.NAJ.0000434169.53750.14.

29. Whisman MA. Loneliness and the metabolic syndrome in a populationbased sample of middle-aged and older adults. Am Psychol Assoc. 2010;29(5):550-4.

30. Keele-Card G, Foxall MJ, Barron CR. Loneliness, depression, and social support of patients with COPD and their spouses. Public Health Nurs. 1993;10(4):245-51.

31. Kara M, Mirici A. Loneliness, depression, and social support of turkish patients with chronic obstructive pulmonary disease and their spouses. J Nurs Sch. 2004;36(4):331-6.

32. O'Súilleabháin PS, Gallagher S, Steptoe A. Loneliness, living alone, and all-cause mortality\#: the role of emotional and social loneliness in the elderly during 19 years of follow-up. Psychosom Med. 2019;81(6):521-6. https://doi.org/10.1 097/PSY.0000000000000710

33. Shankar A, McMunn A, Demakakos P, Hamer M, Steptoe A. Social isolation and loneliness: prospective associations with functional status in older adults. Health Psychol. 2017;36(2):179-87.
34. Rantakokko M, Iwarsson S, Vahaluoto S, Portegijs E, Viljanen A, Rantanen T. Perceived environmental barriers to outdoor mobility and feelings of loneliness among community-dwelling older people. J Gerontol A Biol Sci Med Sci. 2014;69(12):1562-8.

35. Pinel Zafra M, Rubio Rubio L, Rubio Herrera R. Un instrumento de medición de soledad social: escala ESTE II [internet]. Granada: Universidad de Granada; 2009. Disponible en: http://envejecimiento.csic.es/docu mentos/documentos/rubio-soledad-est e2.pdf.

36. Guerrero Ceh JG, Can Valle AR, Morales LM. La intervención gerontológica en un establecimiento de asistencia social permanente privado: identificación de los beneficios que aporta a las personas mayores residentes. Rev Iberoam Ciencias Salud. 2018;7 (14). https://doi.org/10.2 3913/rics.v7i14.73

\section{Notas}

Conflictos de interés: los autores manifiestan no tener conflictos de interés. 